\title{
Reduction of Neurite Outgrowth in a Model of Glial Scarring following CNS Injury Is Correlated with the Expression of Inhibitory Molecules on Reactive Astrocytes
}

\author{
Robert J. McKeon, ${ }^{1}$ Rebecca C. Schreiber, ${ }^{1}$ John S. Rudge, ${ }^{2}$ and Jerry Silver ${ }^{1}$ \\ 'Department of Neurosciences, Case Western Reserve University, Cleveland, Ohio 44106 and ${ }^{2}$ Regeneron \\ Pharmaceuticals Inc., Tarrytown, New York 10591
}

The extracellular matrix (ECM) molecules chondroitin-6-sulfate proteoglycan (CS-PG) and cytotactin/tenascin (CT), present on subpopulations of astroglia or their precursors during development, can inhibit neurite outgrowth in vitro. However, it is not known whether these molecules are expressed within the mature CNS following injury, where they could contribute to regenerative failure. Thus, the expression of various ECM molecules that affect axon growth was examined in areas of reactive gliosis caused by implanting a piece of nitrocellulose into the cortex of neonatal and adult animals. The expression of these molecules was compared to the amount of neurite outgrowth that occurred in vitro when the damaged CNS tissue from animals of various ages was removed intact and used as a substrate in explant culture. The results demonstrate that the growth-promoting molecules laminin, collagen type IV, and fibronectin were present around the implant in all experimental groups. In comparison, CS-PG and CT were present within and around the area of the lesion only in adult animals. In vivo, these molecules were colocalized with intensely glial fibrillary acidic protein (GFAP)-positive astrocytes in and immediately adjacent to the scar, but not with other equally intensely GFAPpositive astrocytes in the cortex away from the site of injury. CT and CS-PG were present in gray matter areas of the cortex that had been directly damaged during the implant procedure and in the corpus callosum when lesioned during implantation. In vitro, the glial tissue removed from the lesion site of neonatal animals supported neurite outgrowth, while scars removed from adult animals did not. The inability of the adult glial scar tissue to support neurite outgrowth was best correlated with the expression of CS-PG and CT, suggesting that these molecules may be involved in limiting the growth of regenerating axons in the CNS after injury.

\footnotetext{
Received Feb. 27, 1991; revised May 17, 1991; accepted May 29, 1991.

We thank Dr. Kathryn Crossin for the generous gift of the cytotactin antibody and Dr. Michiko Watanabe for the gift of the antibody to NCAM. We also thank Catherine Doller for her excellent technical help with the electron microscopy and photography. This work was supported by NS 25713, the Daniel Heumann Fund for Spinal Cord Research, and the Brumagin Fund for Spinal Cord Research. R.J.M. was supported by a Developmental Neurobiology Training Grant T32NS07118.

Correspondence should be addressed to Jerry Silver, Ph.D., Department of Neurosciences, Case Western Reserve University, 2040 Adelbert Road, Cleveland, $\mathrm{OH} 44106$.

Copyright (C) 1991 Society for Neuroscience $0270-6474 / 91 / 113398-14 \$ 05.00 / 0$
}

It is now well established that most axons within the adult vertebrate CNS fail to regenerate following injury (Ramon y Cajal, 1928; Clemente, 1955; Puchala and Windle, 1977; Reier and Houle, 1988). Regenerative failure may be due, at least in part, to environmental constraints within the tissue surrounding damaged axons since, given an appropriate substrate, injured axons can grow for considerable distances (Aguayo et al., 1982). One crucial extrinsic constraint may be the formation of a glial scar (Reier et al., 1983), which consists largely of a dense meshwork of hypertrophied astrocytic processes and their associated matrices (Reier and Houle, 1988; Maxwell et al., 1990a,b).

While it is likely that the density or the three-dimensional geometry of the glial scar creates a mechanical barrier that limits axon growth, recent evidence suggests that changes in the molecular properties of reactive astrocytes associated with a scar may also play a role in regenerative failure (Rudge and Silver, 1990). Rudge and Silver (1990) examined the growth of hippocampal neurons on nitrocellulose filters that had been implanted into the cerebral cortex of neonatal or adult animals and then, after various lengths of time, removed and placed in explant culture. It was demonstrated that significantly less neurite outgrowth occurred on explants removed from adult animals as opposed to neonates. Since, in this in vitro model, neurite outgrowth was inhibited on a two-dimensional surface, the data suggest that factors other than, or in addition to, mechanical constraints may restrict the ability of neurites to grow on a substrate of reactive astrocytes obtained from a site of injury in adult animals.

Another factor that could limit axon regeneration in the area of a lesion is the absence or limited expression of growth-promoting molecules (Snider and Johnson, 1989). Indeed, a decreased expression of adhesive extracellular matrix (ECM) or cell-surface molecules, that is, NCAM and HNK-1, has been shown to occur on the surface of astrocytes as they mature (Smith et al., 1990). However, while a diminished expression of these ligands may limit the amount of neurite outgrowth in vitro, it is still unclear whether a decrease in growth-promoting molecules alone is capable of limiting regeneration after injury within the mature CNS.

Alternatively, axon inhibitory molecules on glia could also diminish regeneration. Schwab and Caroni (1988) have shown that two intrinsic membrane proteins associated with mature oligodendrocytes limit neurite outgrowth in vitro (Caroni and Schwab, 1988a,b) and in vivo (Schnell and Schwab, 1990). However, while it is clear that oligodendrocytes can inhibit neurite outgrowth, it is not known whether molecules that restrict axon 
outgrowth are similarly expressed by other glial cells, specifically reactive astrocytes in gray and white matter regions. Along these lines, two molecules, cytotactin/tenascin (CT) (Grumet et al., 1985; Kruse et al., 1985; Hoffman et al., 1988; Crossin et al., 1989; Steindler et al., 1989; Prieto et al., 1990) and a chondroitin sulfate/keratan sulfate proteoglycan (CS/KS-PG) (Snow et al., $1990 \mathrm{~b}$ ), located on astrocytes in normal boundary regions of the developing embryo, have been shown to limit axon outgrowth in vitro. Substrates of CT (Crossin et al., 1990; Faissner and Kruse, 1990) or chondroitin-6-sulfate proteoglycan (CS-PG, Snow et al., 1990a) inhibit the growth of embryonic neurons in vitro when in sufficient quantity relative to the concentration of growth-promoting molecules. Additionally, in monolayer culture, neurites will avoid a specific subtype of astrocyte that expresses elevated levels of CT but will grow on other astrocytes that express low levels of this molecule on their surface (Grierson et al., 1990). Together, these data suggest that $\mathrm{Cl}$ and, especially, $\mathrm{CS} / \mathrm{KS}-\mathrm{PG}$ are potent inhibitors of neurite outgrowth in vitro.

While the in vivo expression of CT and CS/KS-PG during CNS development is becoming well documented, the expression, or reexpression, of these molecules by astrocytes following injury and their possible role in regenerative failure are still unknown. In the present study, we sought to examine the presence of several putative inhibitory molecules in an area of glial reactivity caused by implanting a piece of nitrocellulose into the cerebral cortex of neonatal and adult rats. The results demonstrate that the expression of the putative inhibitory molecules CS-PG and CT on astroglia is markedly increased in the area of the lesion only in animals implanted as adults. The relative expression of these molecules also differs depending on the length of time postimplantation and, importantly, the placement of the lesion. When the lesion is confined to the gray matter of the cortex, these molecules are present within or immediately adjacent to the filter implant. When the placement of the filter also damages the white matter of the corpus callosum, these molccules are reexpressed in the callosum, extending across the midline well away from the implant. In these animals, staining is limited to the damaged portion of the corpus callosum and is not present in the vicinity of the terminal arbors of damaged axons in the gray matter of the hemisphere contralateral to the implant. Finally, the expression of these putative inhibitory molecules is correlated with a decreased amount of neurite outgrowth from chick retinal ganglion cells grown in vitro on nitrocellulose implants removed from adult animals when compared to neonatal animals. These data suggest that certain subpopulations of reactive astrocytes, in addition to forming physical barriers, may limit axonal elongation in vitro and in vivo by expressing molecules that restrict neurite outgrowth.

\section{Materials and Methods}

Animals. Four groups of Sprague-Dawley rats (Zivic Miller) were used in the present study. The first group consisted of newborn rat pups in which lesions were made by inserting a piece of boiled Millipore filter paper into the cerebral cortex on postnatal day $0(\mathbf{P}-0)$ and allowed to survive for $6 \mathrm{~d}$. The second group consisted of rat pups that were implanted on P-0 but allowed to survive for $30 \mathrm{~d}$. The third group consisted of rats that were implanted on P-30 and allowed to survive for $10 \mathrm{~d}$. Finally, the fourth group consisted of rats that were implanted on P-30 and allowed to survive for $30 \mathrm{~d}$. These ages and survival times were chosen in order to assess the response of host glia to a lesion made before or after the critical period described by Smith et al. (1986). Additionally, the long-term response of glial cells to CNS lesions created before but analyzed after the onset of the critical period could be ex- amined. Twenty animals with well-placed implants in each of the first three groups were processed for immunocytochemistry, while 10 animals were included in the fourth group. Additionally, 10-15 well-placed filters were removed from animals in each of the first three groups and placed in culture for use as a substrate in neurite outgrowth assays (see below).

Surgery. In the present study, pieces of Millipore filter were implanted into the cerebral cortex according to the method of Rudge et al. (1989). Briefly, both groups of P-0 animals were anesthetized on ice. An incision was made on the dorsal surface of the head, and the skin was retracted. The skull was cleaned of the periosteum, and a small rectangular opening was carefully made through the skull. Next, a $2 \mathrm{~mm} \times 2 \mathrm{~mm}$ piece of nitrocellulose filter (Millipore Corp.) was implanted into the cerebral cortex. The incision was closed and the animal returned to its cage.

Similarly, the P-30 animals were anesthetized with Rompun and ketamine $(0.12 \mathrm{cc} / 100 \mathrm{gm}$, i.p.). A midline incision was made through the scalp, and the skin was retracted laterally. After the periosteum was cleaned from the skull, a rectangular window was drilled over the right cerebral cortex. An incision was made in the cortex using a \#11 scalpel blade, and a $3 \mathrm{~mm} \times 3 \mathrm{~mm}$ Millipore filter was inserted. After all bleeding was controlled, the skin was sutured closed and the animal was returned to its cage.

Immunocytochemistry. After the appropriate time interval, both neonatal and adult animals were deeply anesthetized with an overdose of chloral hydrate and perfused with $4 \%$ paraformaldehyde. The brains were posifixed for $4 \mathrm{hr}$ in the same fixative and then cryoprotected with sucrose. Ten-micrometer sections were cut on a cryostat and thawed onto gelatin-coated slides. Sections were then reacted with one of the following antibodies overnight in PBS: anti-laminin (anti-LN; Bethesda Research Laboratories) or anti-glial fibrillary acidic protein (anti-GFAP; Accurate Chemical), each diluted 1:500, or anti-collagen type IV (Southern Biotechnology), anti-fibronectin (anti-FN; Chemicon), or anticytotactin (a generous gift from Dr. K. Crossin, Rockefeller University), each diluted 1:200. Additionally, selected sections were either incubated overnight with a monoclonal antibody, CS-56 (ICN ImmunoBiologicals; diluted 1:25), which recognizes the chondroitin-6-sulfate proteoglycan (CS-PG) species, or for $1 \mathrm{hr}$ with a monoclonal antibody, 3B3 (ICN ImmunoBiologicals, 1:100), which recognizes CS-PG following a $1 \mathrm{hr}$ incubation in $0.1 \mathrm{U} / \mathrm{ml}$ chondroitinase avidin-biotin complex (ABC) al $37^{\circ} \mathrm{C}$. Finally, selected sections were double labeled with mouse anti-GFAP (Boehringer Mannheim) and rabbit anti-CT in order to determine the cellular localization of this inhibitory molecule.

After the primary antibody was removed and the tissue washed in buffer, the sections were then incubated in an appropriate biotinylated secondary antibody $(1: 100)$ for $1 \mathrm{hr}$. Following removal of the secondary antibody, the tissue was washed and then incubated for $30 \mathrm{~min}$ in strepavidin conjugated with Texas red (1:100; Amersham). The sections were rinsed and coverslipped in Citifluor (Citifluor Ltd.) and viewed on a Leitz Orthoplan 2 fluorescent microscope. Two sets of controls were used to check the specificity of the antibody staining. First, in order to determine whether the filter implant was binding any of the antibodies nonspecifically, staining was done in control animals $1 \mathrm{hr}$ after they had received a filter implant, before any of the molecules examined could be newly synthesized by the host. Second, the primary antibody was omitted from adjacent slides of all experimental animals. In both types of controls, the staining was negative. Controls for the double-labeling experiments included omitting the primary antibodies or applying each primary sequentially and then reacting them with the inappropriate secondary.

In addition to examining frozen sections of the implant cut in the coronal plane, filters were removed, intact, from both neonatal and adult animals and stained immunocytochemically with antibodies to GFAP, laminin, collagen type IV, CT, and CS-PG, as above. The surface of these filter implants was examined in order to begin to determine the molecular composition of the cells on the filter prior to using them as a substrate for subsequent neurite outgrowth studies, described below.

Electron microscopy. Tissue sections were prepared for electron microscopy by perfusing two animals that had been implanted at P-30 and allowed to survive for $30 \mathrm{~d}$ with $0.5 \%$ glutaraldehyde $/ 2 \%$ paraformaldehyde in $0.15 \mathrm{~m}$ phosphate buffer. The brains were removed, blocked around the filters, and placed into the same fixative overnight. Next, $300 \mu \mathrm{m}$ sections were cut on a Vibratome. These sections were osmicated, dehydrated, and embedded in Spurr's resin. Serial $1 \mu \mathrm{m}$ sections were taken through the implant and stained with toluidine blue. Selected sections were cut on a Riechert ultramicrotome, stained with uranyl 
acetate and lead citrate, and viewed on a Joel $100 \mathrm{CX}$ electron microscope.

In one adult animal, the filter implant was removed $30 \mathrm{~d}$ postimplantation and prepared for EM immunocytochemistry. Briefly, the animal was perfused with $4 \%$ paraformaldehyde, and the implant was removed from the cortex. The implant was washed $3 \times$ in Tris buffer, incubated in $20 \mathrm{~mm}$ lysine in $3 \%$ normal goat serum (NGS) for $30 \mathrm{~min}$, and rinsed in 3\% NGS in Tris buffer. Next, the implant was incubated in anti-CT (1:200) overnight and rinsed. The implant was incubated in a biotinylated goat anti-rabbit secondary (Vector), diluted 1:100, for 1 $\mathrm{hr}$ followed by three rinses in buffer. The implant was reacted with $0.5 \%$ $\mathrm{H}_{2} \mathrm{O}_{2}$ for $10 \mathrm{~min}$, rinsed, treated according to the ABC method (Vectastain) for $30 \mathrm{~min}$, and visualized by incubating the implant in $0.05 \%$ diaminobenzidine, $0.025 \%$ cobalt chloride, and $0.01 \% \mathrm{H}_{2} \mathrm{O}_{2}$ for $10 \mathrm{~min}$. Finally, the implant was postfixed in $2.5 \%$ glutaraldehyde for $3 \mathrm{hr}$, rinsed, osmicated, dehydrated, and embedded. The implants were cut in the coronal plane and viewed as above.

Neurite outgrowth assay. After the appropriate time in three groups of animals ( 6 or $30 \mathrm{~d}$ postimplantation in neonatal animals vs. $10 \mathrm{~d}$ postlesion in adults), the implanted filter was removed from the animal and placed in vitro according to the method of Rudge and Silver (1990). Briefly, animals were overdosed with chloral hydrate and decapitated. The skull was removed, and the implant was located within the gray matter of the cerebral cortex. The overlying tissue was carefully removed and the implant gently lifted from the brain. The implant was then incubated for $30 \mathrm{~min}$ at $37^{\circ} \mathrm{C}$ in calcium- and magnesium-free Hank's balanced salt solution (HBSS) containing $0.02 \%$ EDTA. The implant was then shaken vigorously to remove any loose tissue not intimately associated with the implant, washed, and placed into Dulbecco's modified Eagle's medium (DMEM)-F12 (GIBCO, Grand Island, NY) with $10 \%$ fetal calf serum (FCS). The medium was changed after $24 \mathrm{hr}$, and the implants were maintained in culture for $3 \mathrm{~d}$.

After $3 \mathrm{~d}$ in vitro, chick retinal neurons were dissociated and seeded onto these implants. Chick retinal ganglion cells were chosen for this study in order, first, to determine whether the difference in the amount of neurite outgrowth on similar implants reported by Rudge and Silver (1990) was species specific, since embryonic day 18 (E-18) rat hippocampal neurons were used in their study. Second, we were interested in establishing a baseline for process outgrowth from chick retinal ganglion cells on these implants in order to study further the effects of function blocking chick-specific antibodies to various adhesive molecules.

Chick retinal ganglion cells were prepared by first removing the ncural retina from the eyes of embryonic day 7 White Leghorn chicks (Squire Vallevue Farm, Hunting Valley, $\mathrm{OH}$ ). Next, the retinas were incubated in $0.1 \%$ trypsin in calcium- and magnesium-free HBSS for $30 \mathrm{~min}$. DNAase $(0.2 \mathrm{mg} / \mathrm{ml})$ was added for the last $10 \mathrm{~min}$. Next, the retinas were centrifuged and resuspended in DMEM-F12 with $10 \%$ FCS. The cells were dissociated and counted, and 25,000 cells $/ 16 \mathrm{~mm}$ well were seeded onto the surface of the implant (see above). The ganglion cells and implants were maintained in culture in DMEM-F12 plus N2 supplements (Bottenstein et al., 1979) and $2 \mathrm{~mm}$ pyruvate. After $48 \mathrm{hr}$, the cocultures were fixed with $4 \%$ paraformaldehyde for $30 \mathrm{~min}$, washed in $50 \mathrm{~mm}$ Tris-buffered saline, and prepared for immunocytochemistry.

Quantification of neurite outgrowth. In cocultures of retinal neurons on the filter implants, chick-specific anti-neural cell adhesion molecule (anti-NCAM; gift from M. Watanabe, Case Western Reserve University) was used to visualize retinal neurons on the surface of the implant. The cocultures were incubated overnight in the primary antibody, washed, and incubated with a secondary antibody conjugated to biotin. Next, the implants were incubated in strepavidin conjugated to horseradish peroxidase. Finally, the retinal ganglion cells were visualized by reacting the implants with $0.5 \mathrm{mg} / \mathrm{ml}$ diaminobenzidene and $0.01 \% \mathrm{H}_{2} \mathrm{O}_{2}$ for $15 \mathrm{~min}$. The endogenous peroxidase in the implant was removed by incubating the implants in $0.5 \% \mathrm{H}_{2} \mathrm{O}_{2}$ for $10 \mathrm{~min}$ at $-20^{\circ} \mathrm{C}$ prior to the strepavidin-HRP step. Specificity of the antibody was determined by deleting the primary antibody in control sections. Antibodies to TuJ1 $(1: 100)$ were used to mark and identify retinal ganglion cells. Staining with anti-TuJ 1 confirmed that retinal ganglion cells survived on all three types of implants and showed a similar decrease in the amount of neurite outgrowth on the adult implants.

The outgrowth of chick retinal neurons on the three different types of implants was determined by digitizing the neuritic lengths from camera lucida drawings. Individual neurons with neurites greater than 2 somal diameters were drawn using a camera lucida attachment on a
Wild M20 microscope at $25 \times$ and $40 \times$ magnification. Using these criteria, 60 cells on each type of implant were digitized with a Numonics digitizer. In order to compare outgrowth on the three different types of explant, the lengths were then placed into bins of increasing $100 \mu \mathrm{m}$ increments and graphically represented as a percentage of total number of neurons with neurites of a specific length. Comparison of neurite lengths on the three different types of implants was performed using a Mann-Whitney $U$ test.

\section{Results}

\section{GFAP immunoreactivity}

Neonates. Neonatal animals received implants on P-0 and were allowed to survive for either 6 or $30 \mathrm{~d}$. Immunocytochemical analysis with antibodies to GFAP revealed a marked increase in the number of GFAP-positive processes along the edge and within the implant in both groups of animals (Fig. $1 A, B$ ). Farther away from the implant, there was only a very limited amount of staining beyond the normal distribution of GFAP-positive astrocytes at these time points. Any increases that were present in the cortex away from the implant were very discrete and were associated with areas of the cortex that had been damaged during the implantation procedure.

Adults. Adult animals (P-30) were also implanted with Millipore filters and allowed to survive for either 10 or $30 \mathrm{~d}$. The distribution of GFAP within these two groups of animals revealed an increased number of intensely GFAP-positive astrocytes both in and around the filter (Fig. 1C) when compared to control animals (Fig. 1D). The greatest density of intensely GFAP-positive astrocytes was found immediately adjacent to the filter implant. Additionally, a significant number of labeled astrocytes were present in the cortex surrounding the implant. However, the density of these cells gradually decreased away from the implant, diminishing until it reached control levels. The increased number and intensity, as well as the diffuse location, of GFAP-positive astrocytes were in marked contrast to the limited GFAP staining in the cortex in animals that had received implants as neonates. Similar to the neonates, intensely GFAP-positive processes were often seen extending into the pores of the implant in adult animals (Fig. 1C). Finally, the number and size of the inserted GFAP-positive processes were consistently greater in animals implanted as adults than in the animals implanted as neonates.

\section{Expression of growth-promoting molecules}

Neonates. Staining for axon growth-promoting molecules, that is, LN, FN, and collagen type IV, gave similar results in neonatal animals and revealed two different patterns. As early as $6 \mathrm{~d}$ postlesion, these molecules, exemplified by LN staining (Fig. $2 A$ ), were present either along the edge or as large deposits within the implant. This pattern of staining was consistent in animals killed $30 \mathrm{~d}$ after filter implantation, although the amount of LN present within the filter was qualitatively greater than that in neonatal animals examined after $6 \mathrm{~d}$ (Fig. $2 B$ ). These data suggest that these particular adhesive molecules are expressed in and around the lesion within 1 week following injury and remain present for at least $30 \mathrm{~d}$.

Adults. All three substrate-adhesive molecules examined in this study were present in the adult animals in and around the filter implants. In filters that were well inserted into the cortex, LN and FN were present either on processes that extended into the filter or as large extracellular deposits throughout the filter (Fig. 2C). Alternatively, in adult animals with greater damage, 

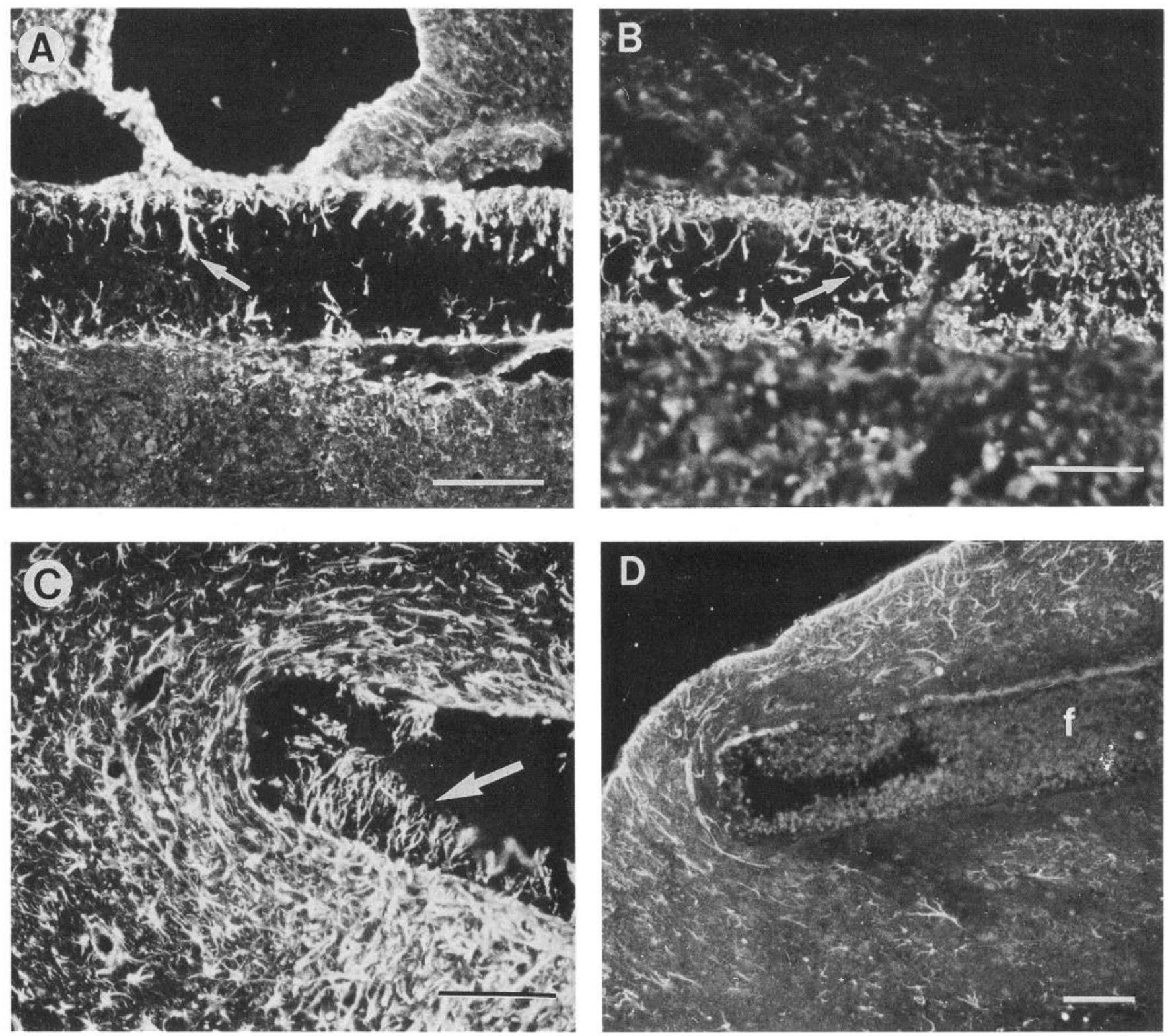

Figure 1. GFAP staining following Millipore filter implantation in neonatal and adult animals. In coronal sections, GFAP-positive astrocytic processes (arrows) are present within the filter in animals implanted on P-1 and killed either $6 \mathrm{~d}(A)$ or $30 \mathrm{~d}(B)$ later. Few, if any, GFAP-positive astrocytes are present in the cortex surrounding the implant. In contrast, there is a dramatic increase in the amount of GFAP staining in the filter and the surrounding cortex in animals implanted on P-30 and allowed to survive for $10 \mathrm{~d}(C)$. Astrocytic processes $(C$, arrow) in these animals are well inserted into the pores of the filter. This staining differs from control animals, who were implanted on P-30 but allowed to survive for only 1 $\mathrm{hr}(D)$. In these animals, only the normal number of GFAP-positive astrocytes are present in the surrounding cortex and are absent from the implant. $f$, filter implant. Scale bars, $200 \mu \mathrm{m}$.

LN, FN, and collagen type IV were located around the periphery of the implant and within sheets of basal laminae (Fig. $3 A-C$ ). $\mathrm{LN}$ and collagen type IV were present both on processes inserted into the filter and in a lacelike network on the filter surface (Figs. $2 D, 3 D$ ). Thus, $\mathrm{LN}, \mathrm{FN}$, and collagen type IV, which have been shown to promote, or at least permit, neurite outgrowth in vitro, are present in or around a filter implant following a cortical lesion in adult animals.

\section{Expression of growth-inhibitory molecules}

Neonates. Immunocytochemical staining for the putative growthinhibitory molecule CS-PG and its ECM ligand, CT, demon- strated, at best, only a limited amount of staining along the edge of the filter at $6 \mathrm{~d}$ postimplantation (Fig. $4 A$ ). Likewise, animals that received Millipore implants on P-0 but were allowed to survive for $30 \mathrm{~d}$ demonstrated little, if any, staining around the implant site. However, staining for these molecules was present in a small triangular region of the subventricular zone along the lateral edge of the lateral ventricle (not shown). The staining in the subventricular zone was identical to that seen in unoperated age-matched control animals. These data suggest that, at two time points following implantation in neonatal animals, CS-PG and CT are either not present or not present in a form recognized by the antibodies used in this study. 

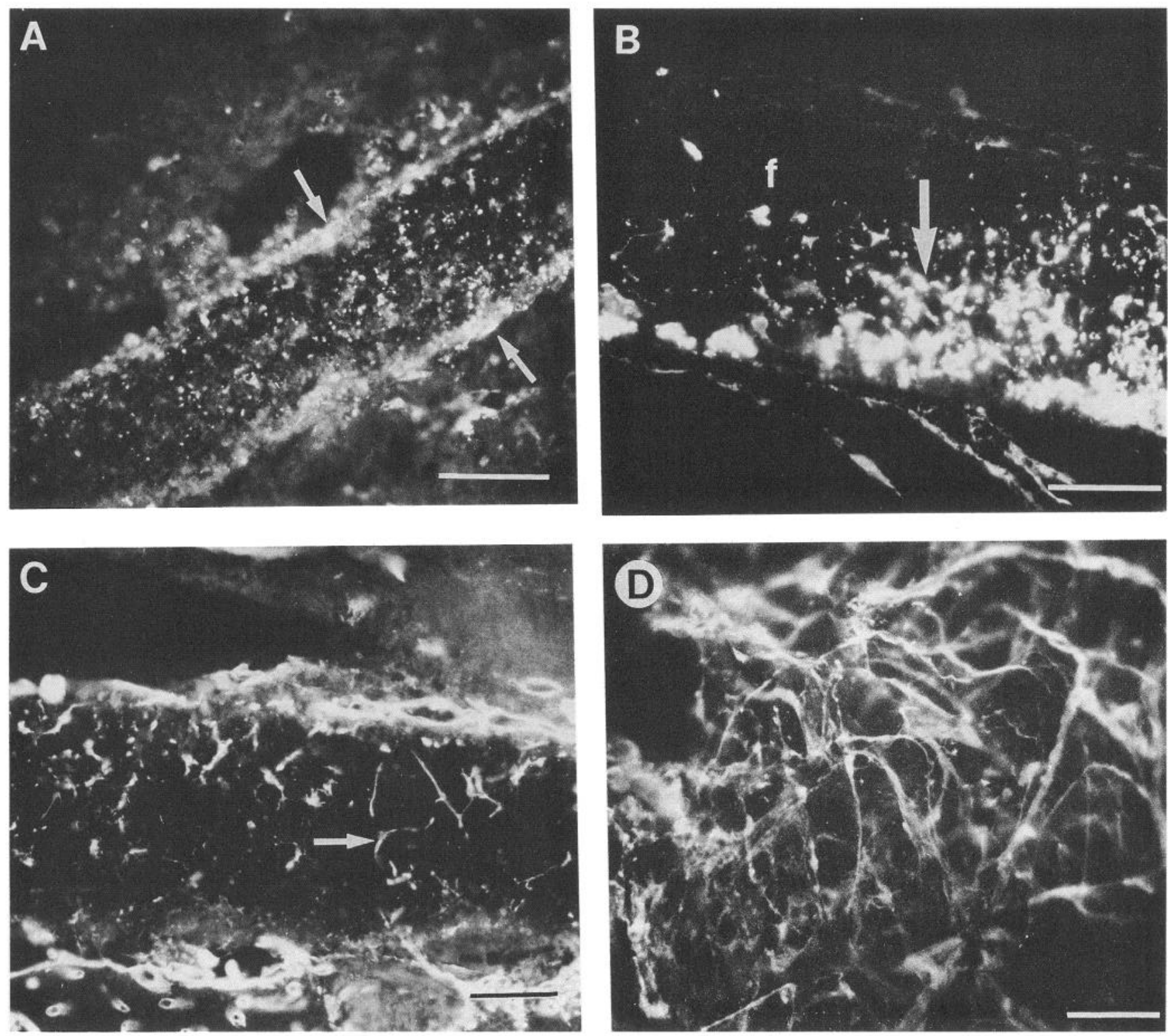

Figure 2. LN immunoreactivity after filter implantation. As early as $6 \mathrm{~d}$ following implantation into neonatal animals $(A)$, $\mathrm{LN}$ is present on the surface of the implant $(A$, arrows $)$ and as punctate deposits within the filter in this coronal section. LN deposition $(B$, arrow) increases in neonatal animals over the course of $30 \mathrm{~d}$ following implantation $(B)$. In adult animals analyzed $10 \mathrm{~d}$ after implantation $(C)$, $\mathrm{LN}$ is present on the surface of the filter and on processes $(C$, arrow) that extend into the filter. When the implant is removed and stained for LN in vitro $(D)$, LN-positive processes can be seen across the surface of the filter. $f$, filter implant. Scale bars: $A$ and $B, 200 \mu \mathrm{m} ; C$ and $D, 100 \mu \mathrm{m}$.

Adults. In comparison to animals implanted as neonates, CSPG and CT were greatly enhanced in animals implanted as adults (Fig. $4 B-D$ ). In each group of adult animals, both molecules were present either within or around the vicinity of the implant, although CS-PG was generally more widely distributed around the filter than CT. The pattern of CS-PG staining often defined the extent of the scar, with staining immediately adjacent to the filter and at the edge of the scar abutting undamaged cortical tissue (Fig. $4 B$ ). Additionally, in filters that were well integrated into the cortex and lacking a basal lamina, CS-PGpositive processes extended into the implant (Fig. $4 D$ ). Similar to the pattern of CS-PG staining, CT, though more limited than the pattern of CS-PG staining, was also present in cells or pro- cesses along the edge of, or inserted into, the filter (Fig. 4C). The staining intensity and distribution of each of these molecules increased over the course of 10-30 d.

Double labeling for GFAP and CT revealed that the GFAPpositive cells and processes within or immediately adjacent to the implant were also positive for CT (Fig. 5). Intensely GFAPpositive astrocytes not associated with the implant were also present in the cortex farther from the filter. However, the GFAPpositive astrocytes away from the implant were not immunoreactive for CT or CS-PG, suggesting that a select population of astrocytes in the adult animal produces CT and CS-PG following this type of injury to the cortex. Specifically, in adult animals, only those astrocytes intimately associated with the 

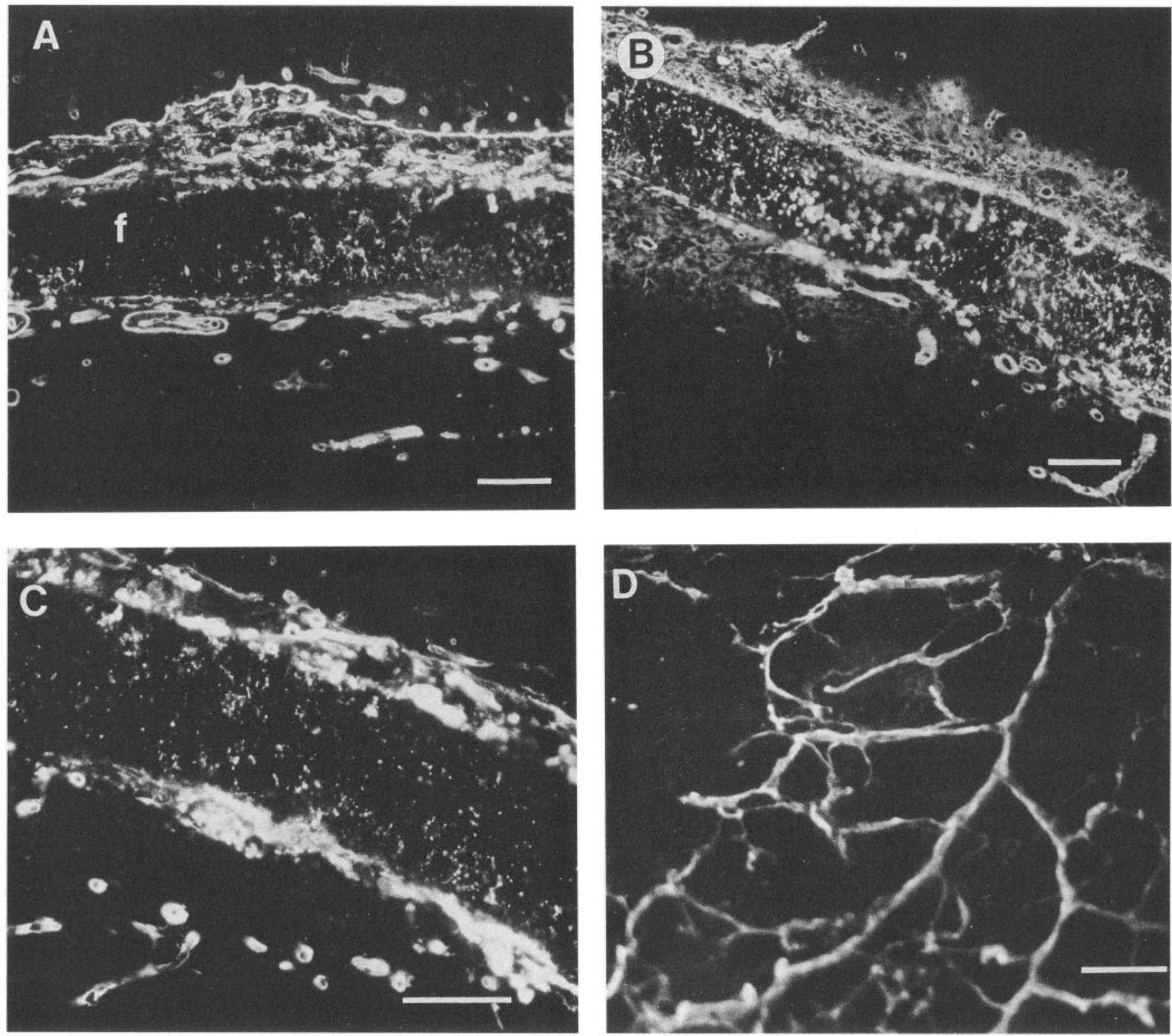

Figure 3. Growth-promoting molecules surrounding the filter implant. In some adult animals analyzed $10 \mathrm{~d}$ after implantation, significant amounts of LN $(A)$, FN $(B)$, and collagen type IV $(C)$ were present around the implant, around blood vessels, and organized into a basal lamina. When the filter was removed and stained in vitro for collagen type IV $(D)$, staining was predominantly along blood vessels that had grown along the surface of the implant. $f$, filter implant. Scale bars, $200 \mu \mathrm{m}$.

filter express these molecules, while those intensely GFAP-positive astrocytes in the surrounding cortex, although "reactive," do not.

Importantly, when implants also involved the white matter (i.e., damaging the corpus callosum), CT and CS-PG staining was present in the callosum, well across the midline (Fig. 6A) and ventrolateral to the implant (Fig. $6 B$ ). The CT and CS-PG staining present in the midline corpus callosum was topographically arranged (Fig. 6A) and corresponded to the portion of the callosum damaged by the implant. While intense CT staining was present immediately around the implant and in the white matter tracts of the callosum at some distance from the implant, no staining was present in the undamaged gray matter surrounding the corpus callosum in the contralateral hemisphere. The observation that staining occurred in areas away from the implant demonstrates that the enhanced expression of CT and CSPG is related to the synthesis or binding of these molecules by astrocytes and not simply reflective of binding of protein by the Millipore filter used in the present study.

$E M$ analysis. In order to examine the nature of the host response to implantation more closely, two animals that received an implant on P-30 were allowed to survive for $30 \mathrm{~d}$ and then were prepared for EM analysis. EM analysis revealed a heavy infiltration of host astrocytes throughout the filter implant (Fig. $7 A, B$ ). Glial processes inserted deep into the filters generally were not surrounded by a basal lamina. Areas of basal lamina were scattered throughout the scarred area, but a continuous basal lamina was not present around astrocytic processes or 

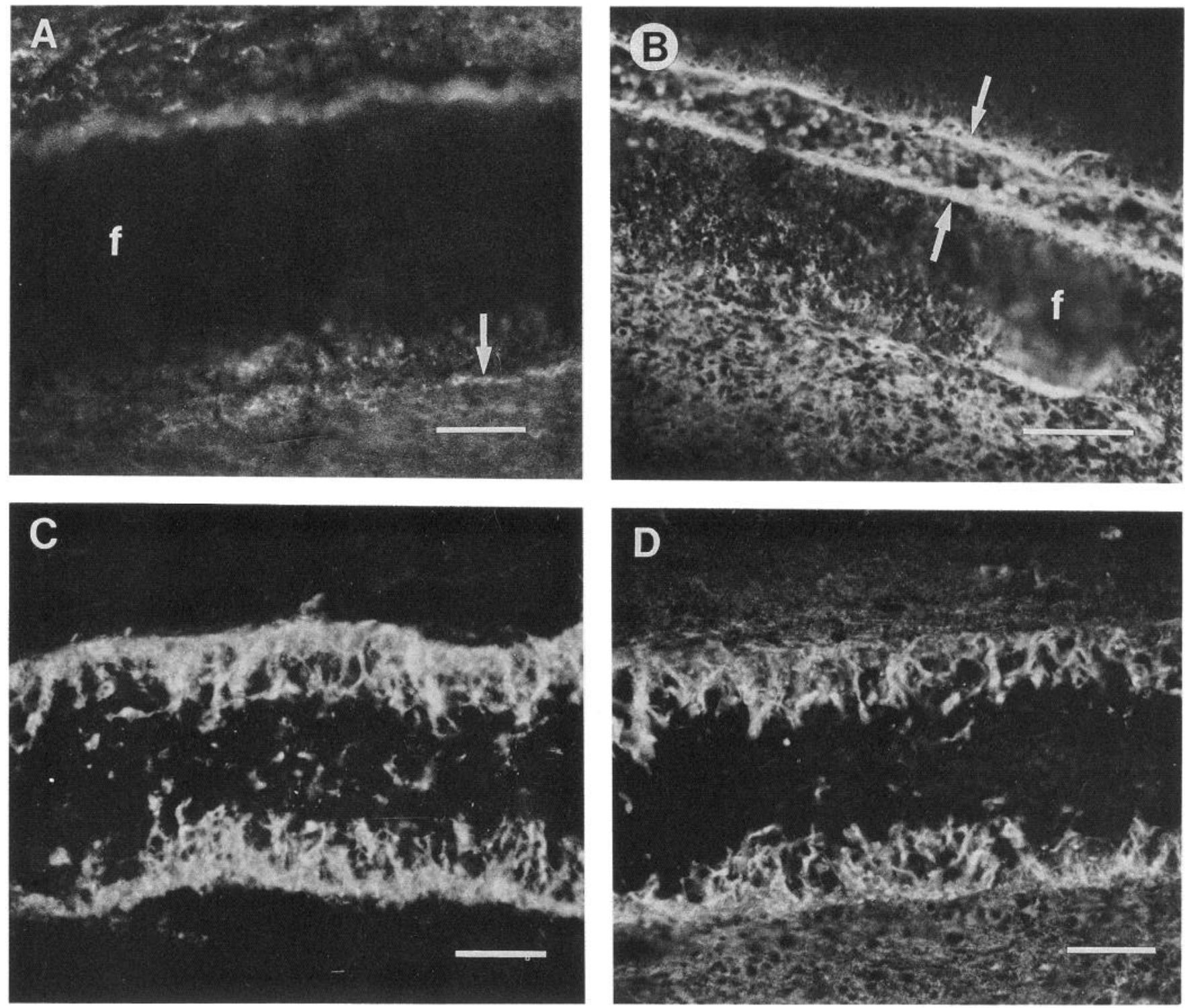

Figure 4. Expression of the putative growth-inhibitory molecules CT and CS-PG following implantation. In neonatal animals analyzed either 6 $\mathrm{d}(A)$ or $30 \mathrm{~d}$ (not shown) following implantation, only a limited amount of CT- $(A$, arrow) or CS-PG-positive staining was present in the area of the implant. In contrast, both molecules were present around the implant in adult animals $10 \mathrm{~d}$ or $30 \mathrm{~d}$ after implantation. In adult animals with a significant amount of tissue damage $(B)$, CS-PG staining was present in layers along the edge of the implant $(B$, arrows) and defined the extent of the scar. In adult animals with well-inserted implants and relatively little associated tissue damage, positive staining for CT $(C)$ and CS-PG $(D)$ was present on processes that extended into the filter implant and was not apparent in cortical tissue surrounding the implant. $f$, filter implant. Scale bars: $A, C$, and $D, 100 \mu \mathrm{m} ; B, 200 \mu \mathrm{m}$.

along the edges of the implant. Few fibroblasts, and no neurons, were present either in or around the filters deep in the brain. Collagen matrices, when present, were limited to areas where fibroblasts were located. Additionally, macrophages could be found throughout the filter implant.

In order to determine the location of at least one of the putative inhibitory molecules of interest, the filter implant was removed from an adult animal $30 \mathrm{~d}$ after implantation and processed for CT EM immunocytochemistry. CT-positive staining was present along the membranes of cells found on the surface of the implant (Fig. 7C) in areas consistently occupied by astroglia in tissue counterstained with uranyl acetate and lead citrate.

Neurite outgrowth studies. E-7 chick retinas were dissociated and seeded onto filter implants that had been removed from neonatal or adult animals at different time points and placed in vitro for $3 \mathrm{~d}$. Neurons and the implants were cocultured for 48 $\mathrm{hr}$, at which time the amount of neurite outgrowth was determined. The surface of the implants was covered with GFAPpositive astrocytes that were similar in viability and morphology to those on filter implants reported by Rudge and Silver (1990).

The neurite outgrowth from chick retinal neurons seeded onto the surface of the filter implants removed from neonatal animals after $6 \mathrm{~d}$ in situ was characterized by a single axon-like projection with a few short neurites also extending from the cell body (Fig. $8 A$ ). In some cases, large palmate growth cones were evident (Fig. $8 D$ ), while in other cases irregular blebs were seen along the length of the neurite. The morphology of the retinal neurons 

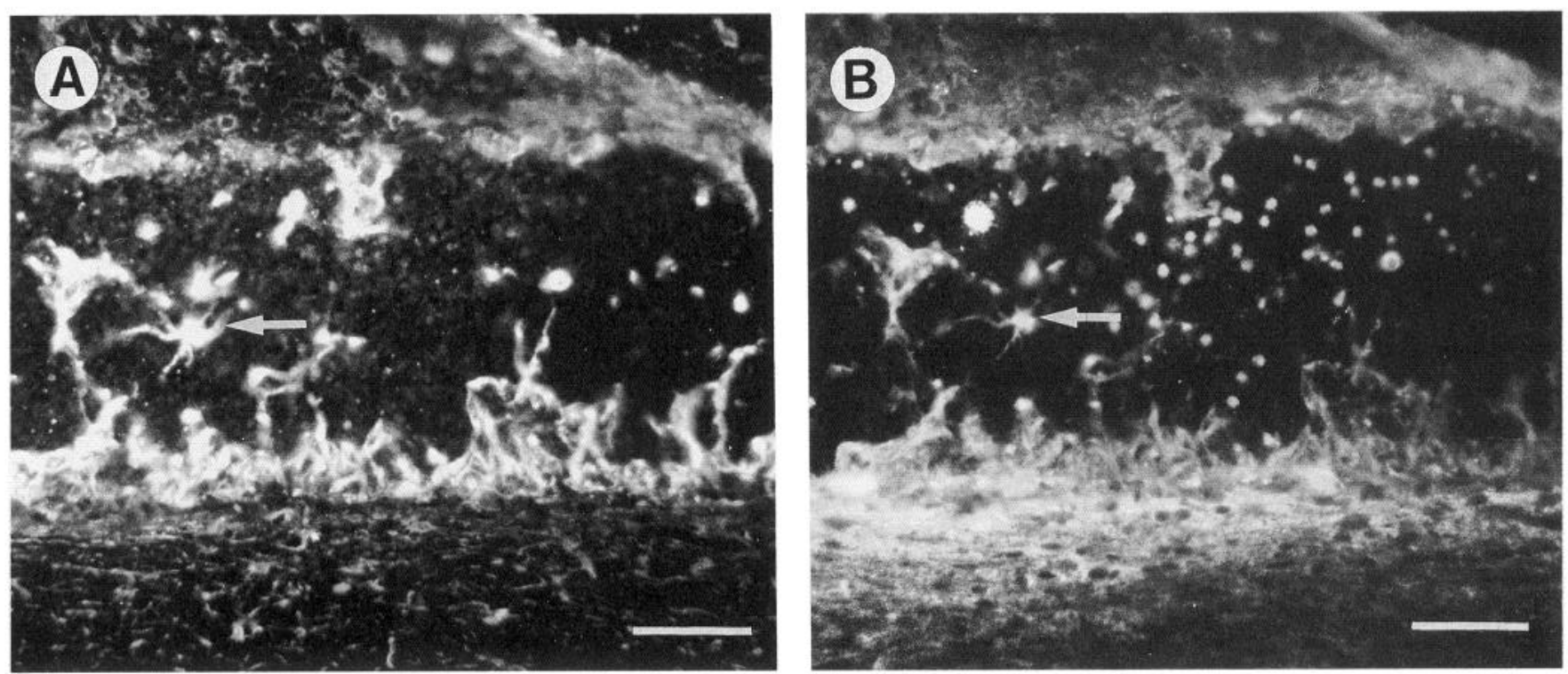

Figure 5. Expression of inhibitory molecules by astrocytes associated with the filter implant. In adult animals, coronal sections through the implant were double labeled for GFAP $(A)$ and CT $(B)$. Astrocytes along the edge and within (arrows) the implant and astrocytic processes that extend into the filter were all positive for cytotactin. Scale bars, $100 \mu \mathrm{m}$.

seeded onto filters removed $30 \mathrm{~d}$ after being implanted into neonates was similar to neurons grown in vitro on the $6 \mathrm{~d}$ implants, although in these the length of the neurites was approximately half the average neuritic length seen on the $6 \mathrm{~d}$ implants (Fig. 8B).

The morphology of retinal neurites grown on filters implanted into adult animals and removed after $10 \mathrm{~d}$ was in marked contrast to neurons present on the $6 \mathrm{~d}$ or $30 \mathrm{~d}$ filters in vitro. The surface of the filters removed from adult animals contained astrocytes that were both highly GFAP-positive and significantly hypertrophied compared to filters removed 6 or $30 \mathrm{~d}$ after implantation into neonates. On these astroglial scars, few neurons possessed long axon-like projections. Rather, the majority of the retinal neurons possessed short primary neurites plus many additional small processes extending from the major neurite (Fig. $8 C$ ).

The differences between the amount of retinal neuronal outgrowth on filter implants removed from neonates after $6 \mathrm{~d}$ or from adults after $10 \mathrm{~d}$ were compared from camera lucida representations (Fig. $8 E$ ). Values from all three groups were represented as the percentage of neurons with neurites greater than the length shown on the $x$-axis (Fig. 9). Approximately $70 \%$ of neurons grown on the $6 \mathrm{~d}$ implants had a neurite greater than $200 \mu \mathrm{m}$. In comparison, $40 \%$ of the neurites on the 30 dimplants
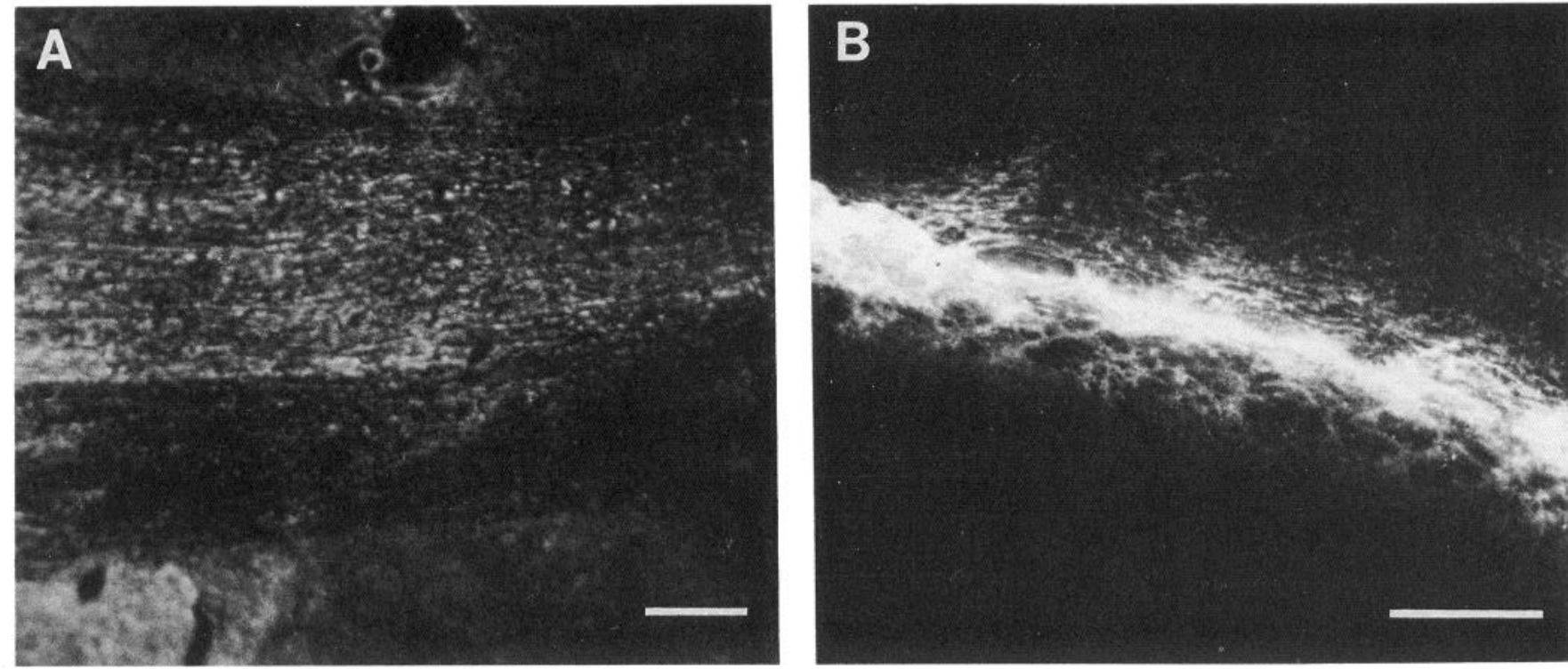

Figure 6. Expression of inhibitory molecules in white matter tracts following injury. When the filter implant damages the corpus callosum, CT and CS-PG are expressed in areas well away from the implant. CT staining $(A)$ is present in a topographical arrangement associated with damage to axons in the dorsal half of the corpus callosum. Staining for CS-PG $(B)$ extends for a significant distance in the callosum ventrolateral to the implant. Scale bars, $200 \mu \mathrm{m}$. 



Figure 7. EM analysis of filter implants in adult animals. A, Astrocytic processes (arrows) grow for significant distances within the pores of the filter implant $(4500 \times)$. Astrocytic processes can be identified more clearly at higher magnification in $B(11250 \times)$. Implants were removed from adult animals after $30 \mathrm{~d}$ and processed for EM immunocytochemistry for CT and CS-PG $(C)$. CT-positive immunoreactivity $(C$, arrows) is present along the border of cells located on the surface of the implant shown in this electron micrograph, which was not otherwise stained with heavy metals $(20,000 \times) . f$, filter implant. 

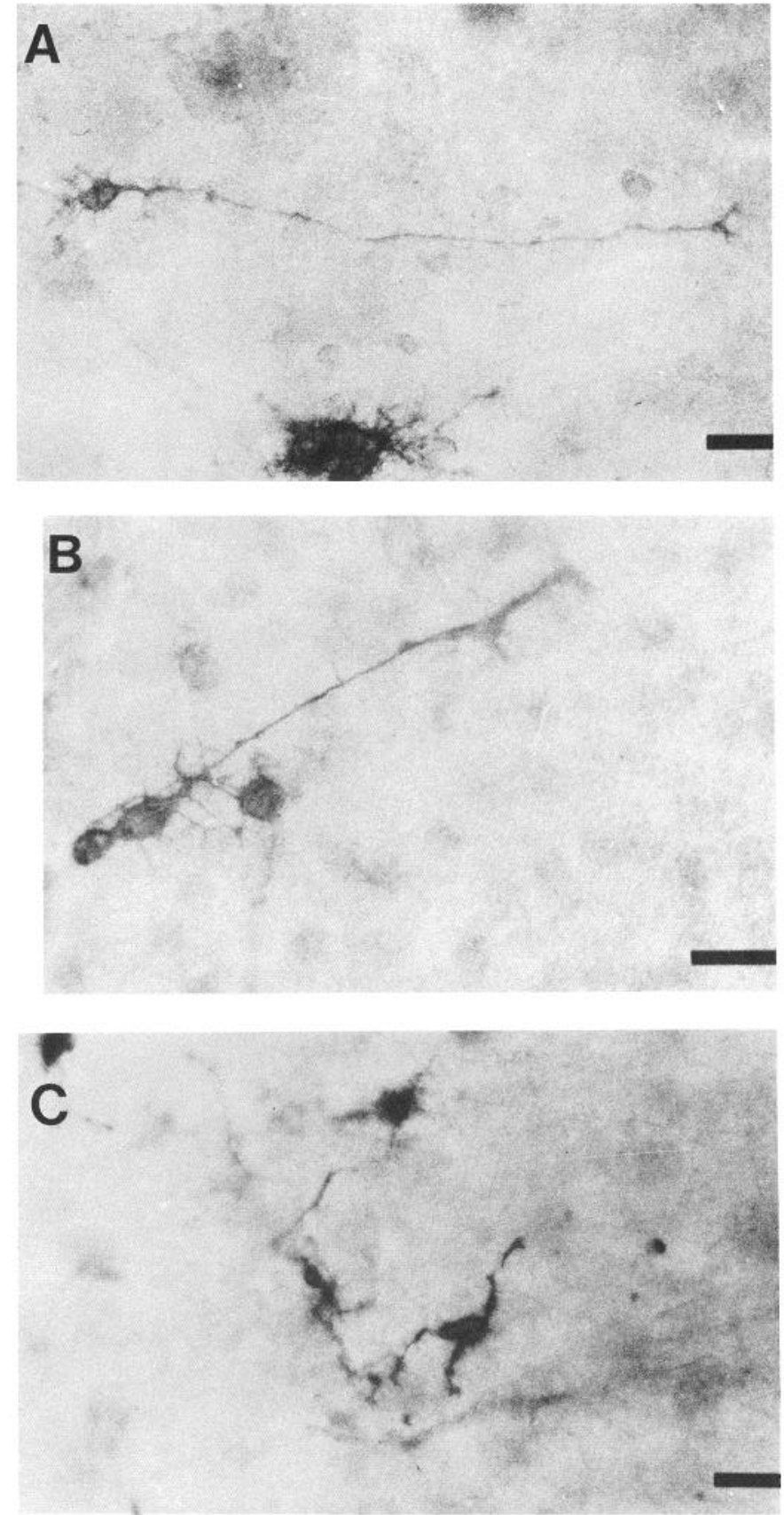

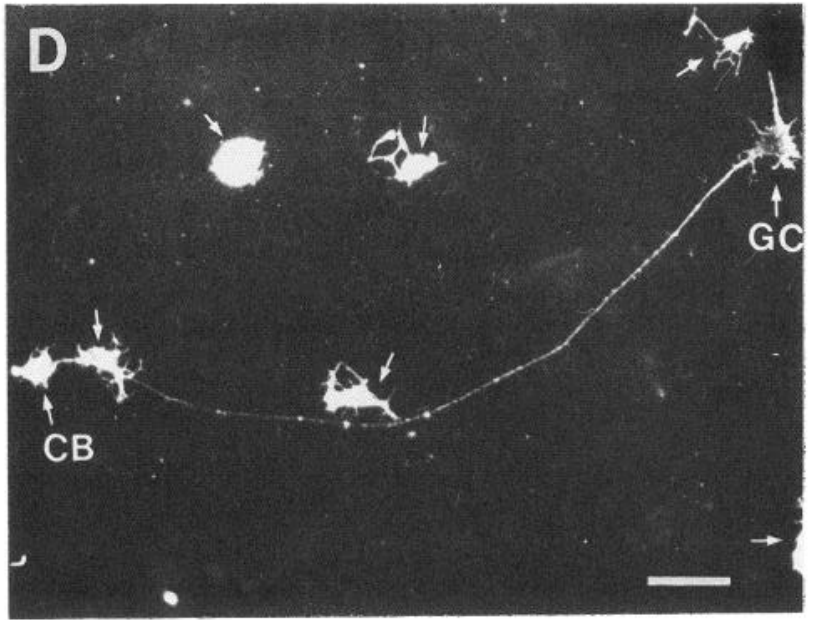

A

$$
1
$$

B

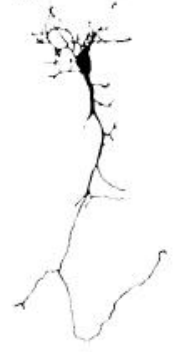

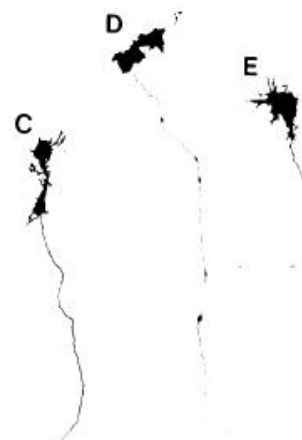

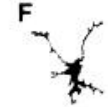

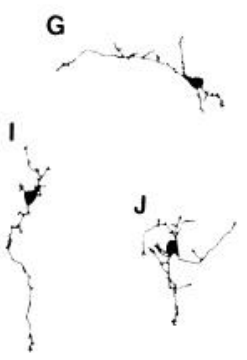
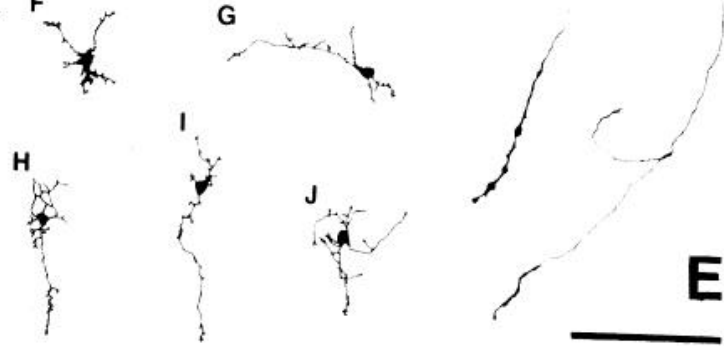

Figure 8. Growth of chick retinal ganglion cells on the surface of Millipore filters implanted into neonatal or adult animals. A significant amount of neurite outgrowth occurs on filters removed from neonates after $6 \mathrm{~d}(A)$. The amount of neurite outgrowth on filters removed from animals implanted as neonates and removed after $30 \mathrm{~d}(B)$ is decreased when compared to the amount of neurite outgrowth shown in $A$. Neurite outgrowth is dramatically reduced on filters removed from adult animals $10 \mathrm{~d}$ after implantation $(C)$. In some cases, on 1-6 day implants $(D)$, large palmate growth cones $(G C)$ were evident. Small arrows in $D$ indicate labeled neurons that were not counted since they did not possess a process greater than 2 somal diameters. $E$, Camera lucida representations of E-7 chick retinal ganglion cells grown, in vitro, on the surface of filter implants removed from neonates after $6 \mathrm{~d}$ (cells $A-E$ ) or adults after $10 \mathrm{~d}$ (cells $F-J$ ). Scale bars: $A-D, 20 \mu \mathrm{m} ; E, 10 \mu \mathrm{m}$.

had a neurite of at least $200 \mu \mathrm{m}$, while only $10 \%$ of the neurites grown on the filters removed from adult animals $10 \mathrm{~d}$ after implantation were as long. The mean value for the length of the longest neurite on the neonatal $6 \mathrm{~d}$ implants after $48 \mathrm{hr}$ was 359 $\mu \mathrm{m}$, with one neurite attaining a length of $1215 \mu \mathrm{m}$. Mean neurite outgrowth on filters implanted into neonatal animals and maintained for $30 \mathrm{~d}$ was $178 \mu \mathrm{m}$, while the longest neurite attained a length of $675 \mu \mathrm{m}$. This represents a $51 \%$ reduction in the mean neurite outgrowth when compared to the neonatal implants maintained for $6 \mathrm{~d}$ in situ. The mean length of neurite outgrowth on filters implanted into adult animals and removed after $10 \mathrm{~d}$ was $85 \mu \mathrm{m}$, with the longest neurite being $475 \mu \mathrm{m}$. This represents a $76 \%$ reduction in neurite outgrowth compared to the amount of growth on implants removed from 6-d-old neonates and a $47 \%$ decrease compared to the amount of growth on implants placed into the cortex of neonatal animals and 

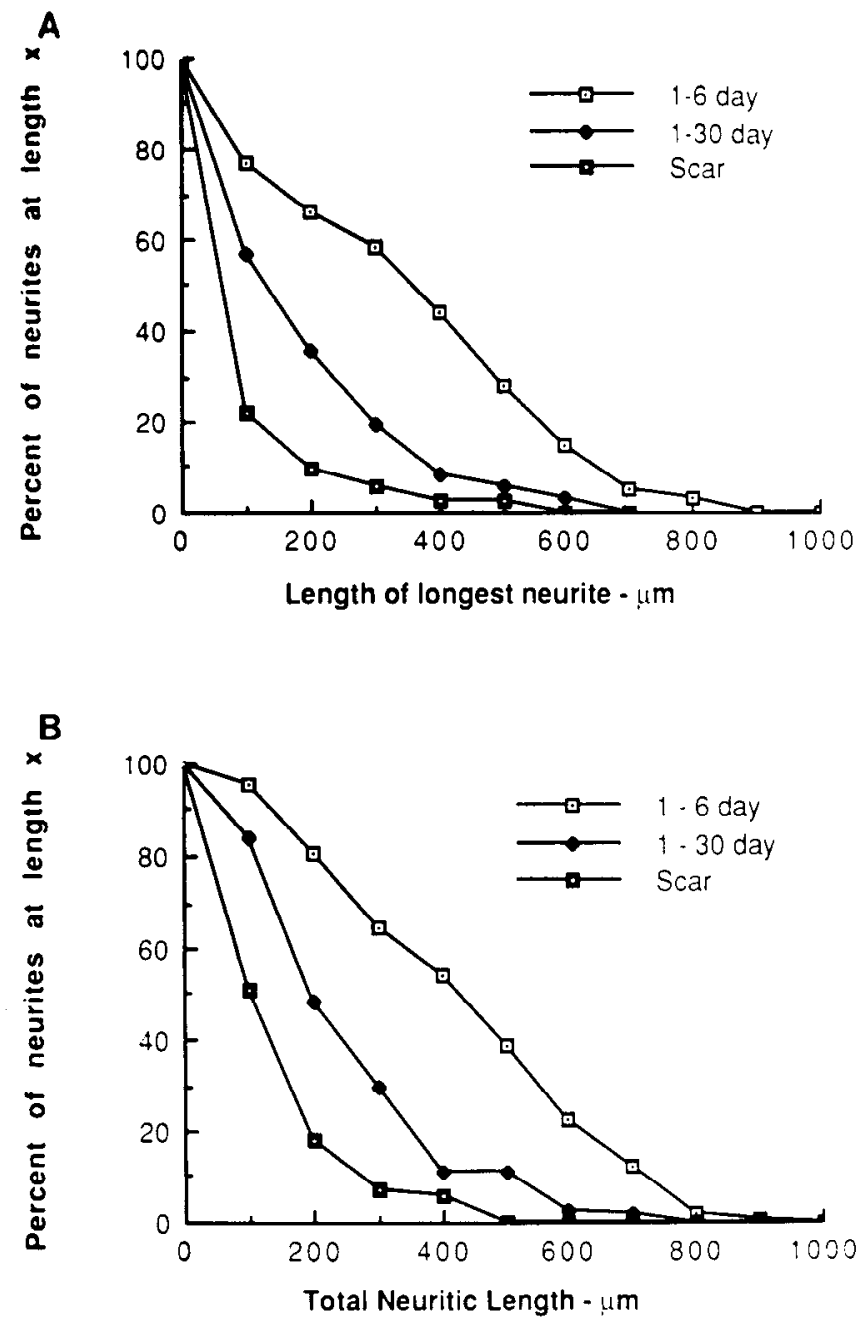

Figure 9. Comparison of the length of E-7 chick retinal ganglion cells on implants removed from neonates after $6 \mathrm{~d}(1-6$ day), $30 \mathrm{~d}(1-30$ day), or adults after $10 \mathrm{~d}$ (Scar). Distributions of both the length of the longest neurite $(A)$ and the total neuritic length $(B)$ were plotted as a percentage of neurites (vertical axis) at a specific length measured in microns (horizontal axis). In $A$, significant differences in neurite outgrowth were observed between groups: $1-6 d / 1-30 d, p<0.001 ; 1-6$ $d /$ scar, $p<0.001 ; 1-30 d /$ scar, $p<0.001$. In $B$, significant differences were also noted in the total amount of neurite outgrowth between groups: $1-6 d / 1-30 d, p<0.001 ; 1-30 d /$ scar, $p<0.001 ; 1-30 /$ scar,$p<0.001$.

removed after $30 \mathrm{~d}$. The inhibition of neurite outgrowth appears to be dependent upon the presence of living cells on the filter implant since no inhibition was noted when neurons were seeded onto fresh-frozen sections of adult cortical scar tissue (J. S. Rudge and J. Silver, unpublished observations).

In order to determine whether the differences in the length of neurite outgrowth were due to neuritic redistribution, the total length of neurites on cach type of implant was detcrmined. Total neuritic length was $437 \mu \mathrm{m}$ for the $6 \mathrm{~d}$ neonatal implants, 240 $\mu \mathrm{m}$ for the $30 \mathrm{~d}$ neonatal implants, and $132 \mu \mathrm{m}$ in the $10 \mathrm{~d}$ adult implants. These data suggest that the majority of chick retinal ganglion neurite outgrowth is concentrated in the single axon-like projection and that the difference in the amount of neurite outgrowth is not due to neuritic redistribution.

\section{Discussion}

Neurite outgrowth is correlated with the expression of inhibitory molecules

In the present study, immunocytochemical markers were utilized to study the presence of axon growth-promoting versus putative inhibitory molecules in areas of gliosis in vivo. Second, the amount of neurite outgrowth from chick retinal ganglion cells that occurred in vitro on filters removed from the cortex of neonatal or adult animals was also analyzed in relation to the expression of these ECM molecules. By using the filter implant as a substrate, all the cellular and molecular components of the glial reaction to lesioning could be removed from the cerebral cortex of animals of different ages.

Our results demonstrate that the cellular and molecular components of the glial response collected on Millipore filters implanted into the cortex of neonatal animals and removed after $6 \mathrm{~d}$ supported significant process outgrowth from chick retinal ganglion cells. In comparison, the components of a glial scar created in the cortex of adult animals allowed little process outgrowth. These observations corroborate recent findings by Rudge and Silver (1990), who assessed the growth of E-18 rat hippocampal neurons when seeded, in vitro, onto nitrocellulose filters retrieved from the cerebral cortex of either neonatal or adult animals. Thus, the growth characteristics of neurites on implants removed from rats and placed in vitro are not species specific. Additionally, our results showing intermediate levels of axon elongation on implants $30 \mathrm{~d}$ after implantation into neonatal animals are consistent with the in vitro observation by Smith et al. (1990) that the intrinsic ability of astrocytes to support neurite outgrowth diminishes after the second postnatal week. The present data also suggest that the ability of mature cortical glia to support neurite outgrowth, in vivo, changes even more dramatically around a specific time frame in response to direct, penetrating injuries. Possible mechanisms that effect this difference in neurite outgrowth on a substrate of reactive glia include the absence of growth-promoting molecules, the presence of inhibitory molecules, or both.

LN and FN were present in all groups examined, and the relative amount of staining for these ligands increased in each experimental group at the longer time points following filter implantation. Thus, if present in the proper configuration, at least two potential growth-promoting molecules are present in the vicinity of a glial scar. Because LN and FN were present both in and around all implants, the significant decrease in neurite outgrowth on implants removed from adult animals appears to be related more closely to the presence of inhibitory ECM molecules rather than the absence of growth-promoting molecules. These molecules appear to be associated with living cells and ECM since neurite outgrowth is not inhibited on sections of fresh-frozen adult cortical scar tissue where ECM molecules are likely to be solubilized in vitro.

One striking phenomenon that occurred within and adjacent to the glial scar was the age-dependent difference in the expression of both CT and CS-PG in the neonatal versus the adult animals following implantation. Both molecules were only expressed in the area of injury following lesions to the cerebral cortex of adult rats and were, importantly, minimal in younger animals. Indeed, only a limited amount of growth occurred in vitro on glial scars removed from adult animals. These data suggest that these molecules may be capable of limiting axon growth despite the continued presence of the substrate-adhesive 
molecules LN and FN both in and around the filter implant at all time points. While CT (Crossin et al., 1990) and, especially, CS-PG (Snow et al., 1990a) can limit neurite outgrowth in vitro individually, the combination of these two molecules with each other or with other still unidentified molecules may be responsible for the inhibitory effect. Finally, experiments are currently in progress to determine whether the enzymatic removal of the glycosaminoglycan side chains (see Snow and Silver, 1990) will allow for enhanced growth of neurites on scars retrieved from adult animals.

\section{Expression of inhibitory proteoglycans and CT during development and after injury}

The expression of sulfated proteoglycans and CT during the development of the nervous system has received an increased amount of attention recently (Verna et al., 1989; Steindler et al., 1990; Tosney and Oakley, 1990; Perris et al., 1991). While the function of CS-PG in isolation appears to be consistently inhibitory (Perris and Johansson, 1990), the role of CT remains controversial. Studies have shown that the distribution of CT coincides with the expression of FN (Epperlein et al., 1988) and is associated with neural crest cell migration (Mackie et al., 1988). Alternatively, other studies (Newgreen et al., 1986, 1990; Tan et al., 1987) have examined the migration of neural crest cells in relation to the expression of both CT and CS-PG in boundary regions. Migrating crest cells avoided areas where the expression of these two molecules was elevated, suggesting that the coupled expression of CT and CS-PG has a restrictive effect on neural crest cell migration. However, it has been reported that CT used alone as a substratum can sometimes promote, and at other times inhibit, cell movement (Chiquet, 1989).

In addition to their effect on cell migration, both KS-PG (Snow et al., 1990b) and CS-PG (M. Gonzales and J. Silver, unpublished observations) are present on roof plate glia in the CNS of the developing embryo. The pattern of distribution of these molecules changes in the dorsal midline during development, and it has been suggested that sulfated PGs in this region may serve as a chemical barrier to growing axons in order to help direct them to their appropriate targets (Snow et al., 1990b). A number of studies (Steindler et al., 1989; Crossin et al., 1990) have also demonstrated PG-and CT-positive boundaries within the developing mouse CNS, outlining the developing somatosensory cortical barrel fields. The expression of both CT and a proteoglycan that binds to CT (CTB) is altered following selective electrocauterization of the whisker follicles (Crossin et al., 1990), suggesting that in these regions expression is activity dependent. Consistent with these developmental studies, recent in vitro experiments, supportive of a barrier function for these molecules, have demonstrated that CT and CS-PG can serve as inhibitory substrates for the growth of embryonic dorsal root ganglia (Crossin et al., 1990; Snow et al., 1990a), embryonic mesencephalic and hippocampal neurons (Faissner and Kruse, 1990), retinal neurites (Snow and Silver, 1990), and postnatal cerebellar neurons (Faissner and Kruse, 1990). The inhibition on CS-PG could be overcome by enzymatic removal of the glycosaminoglycan side chains from the proteoglycan as well as by increasing the concentration of $\mathrm{LN}$ in relation to the proteoglycan (Snow et al., 1990a). Finally, recent observations in our lab have demonstrated that CT and CS-PG may have inhibitory properties in another nonregenerating system, that is, the dorsal root entry zone (DREZ) of the spinal cord. Although lacking in the DREZ prenatally, CT and CS-PG staining is increased in the dorsal columns and DREZ by $3 \mathrm{~d}$ postnatally. The expression of these molecules is greatly enhanced following root crush after P-3. The changing distribution of CT and CS-PG is in exact relation to the pattern of GFAP-positive astroglia (Pindzola and Silver, 1990). The expression of these putative inhibitory molecules may be involved with the inability of regenerating peripheral fibers to reenter the CNS immediately after this time point, as described by Carlstedt et al. (1987). Taken with the results of the present experiments, these studies suggest that putative inhibitory molecules are present on certain populations of astroglia or their precursors in vivo during development and, importantly, are reexpressed in certain regions of the CNS in adult animals following injury.

\section{Astrocytes as a possible source of inhibitory proteoglycans}

A number of cell types contribute to the formation of a glial scar. EM analysis and in vitro labeling (Rudge et al., 1989) have demonstrated, however, that astrocytes are the main cell type in and around the filter implant. Although a CS/KS-PG, recognized by CAT 301 and VC1.1, has been shown to be concentrated around synapses postnatally (Zaremba et al., 1990), the overall expression of these molecules within the cerebral cortex and spinal cord decreases during the first postnatal week (Crossin et al., 1986; Tan et al., 1987; Prieto et al., 1990). In the present study, we have demonstrated that the expression of both CT and CS-PG increases in the CNS of adult animals after injury. After a gray matter lesion, CT is colocalized with GFAPpositive astrocytes within and immediately adjacent to the filter, suggesting that the subpopulation of astrocytes abutting the injured area is the source of this inhibitory molecule. It has been reported that certain subpopulations of astrocytes express CT (Faissner, 1988; Prieto et al., 1990) and/or CS/KS-PG (Snow et al., 1990b) during development. Additionally, Gallo and Bertolotto (1990) have demonstrated that astrocytes and their progenitor cells, but not oligodendrocytes, can express CS-PG in vitro. CS-PG was present both within the cell and in the culture medium, suggesting that astrocytes are capable of secreting this molecule into the extracellular space (Gallo and Bertolotto, 1990).

Following injury to the corpus callosum, CT and CS-PG, most likely associated with astrocytes, can be seen solely within the tract at some distance from the lesion site within regions where callosal axons were damaged during the implantation procedure. These data suggest that the molecular response of gray versus white matter astrocytes differs following axotomy. Gray matter astrocytes produce CT and CS-PG in response to direct cortical trauma, while white matter astrocytes in certain regions may be capable of producing these molecules following either a direct injury or in response to damage to axons en passant. However, the specific signal that causes the expression of these molecules in white matter regions away from the site of injury remains unknown.

GFAP-positive reactive astrocytes versus GFAP-, CT-, and CS$P G$-positive reactive astrocytes

Following injury to the CNS, astrocytes hypertrophy and increase their expression of GFAP. This process, known as reactive gliosis, contributes to the formation of an astroglial scar around many types of CNS lesions. In the present study, a dramatic increase in GFAP staining took place in the area surrounding and extending from the lesion created by implanting a piece of Millipore. By convention, all intensely GFAP-positive astrocytes, regardless of their proximity to the lesion, would be 
considered "reactive" and, potentially, contribute to regenerative failure, yet it is known that in vitro gray matter is permissive for neurite outgrowth (Savio and Schwab, 1989). Additionally, a good deal of plasticity can occur in gray matter adjacent to cortical injuries (Jenkins and Merzenich, 1987), in hippocampal gray matter after deafferentation (Lynch et al., 1972; Stanfield and Cowan, 1982; McKeon et al., 1989), and in the dorsal horn of the spinal cord after root lcsions (Goldberger and Murray, 1974), suggesting that "reactive" astrocytes not intimately associated with a lesioned area may provide positive tropic and/ or trophic signals for sprouting and synaptic rearrangement. This suggestion is consistent with our observation that the inhibitory molecules examined in the present study are not present in gray matter regions where sprouting is known to occur. Thus, in gray matter, differences in the "reactive nature" of astrocytes may occur based on their proximity to a penetrating lesion. Those astrocytes located around the lesion may be most capable of limiting or preventing axonal regeneration by expressing inhibitory molecules, suggesting that the expression of CT and certain sulfated proteoglycans, rather than GFAP alone, may be more appropriate markers for identifying subpopulations of astrocytes that contribute to regenerative failure in the CNS. This hypothesis is consistent with the observation that the least amount of neurite outgrowth occurred in vitro on filters where the expression of CT and CS-PG was the greatest.

In conclusion, the results of the present experiments demonstrate that both growth-promoting molecules and two putative inhibitory molecules associated with astrocytes are expressed within the gray and white matter of the CNS following injury to adult animals. The expression of CS-PG and CT is correlated with a decreased amount of neurite outgrowth on glial scars in vitro. These data suggest that changes in the relative balance of positive versus inhibitory factors may alter the local environment and, ultimately, influence the amount of regeneration that takes place in the CNS following injury. By determining the interaction between positive versus inhibitory molecules, it may be possible to devise strategies to enhance the growth-promoting components of gliosis while limiting the expression of inhibitory molecules, leading to greater process outgrowth both in vitro and in vivo.

\section{References}

Aguayo A, David S, Richardson P, Bray G (1982) Axonal elongation in peripheral and central nervous system transplants. Adv Cell Neurobiol 3:215-234.

Bottenstein J, Hayashi I, Hutching S, Masui H, Mather J, McClure DB, Ohasa S, Rizzino A, Sato G, Serrero G (1979) Growth of cells in serum free hormone supplemented media. Methods Enzymol 58:94 109.

Carlstedt T, Dalsgaar CJ, Molander C (1987) Regrowth of lesioned dorsal root fibers into the spinal cord of neonatal rats. Neurosci Lett 74:14-18.

Caroni P, Schwab ME (1988a) Two membrane protein fractions from rat central myelin with inhibitory properties for neurite outgrowth and fibroblast spreading. J Cell Biol 106:1281-1288.

Caroni P, Schwab ME (1988b) Antibody against myelin-associated inhibitor of neurite growth neutralizes nonpermissive substrate properties of CNS white matter. Neuron 1:85-96.

Chiquet M (1989) Tenascin/J1/cytotactin: the potential function of hexabrachion proteins in neural development. Dev Neurosci 11:266275.

Clemente CD (1955) Structural regeneration in the mammalian central nervous system and the role of neuroglia and connective tissue. In: Regeneration in the central nervous system (Windle WF, ed), pp 147161. Springfield, IL: Thomas.
Crossin KL, Hoffman S, Grumet M, Thiery J-P, Edelman GM (1986) Site-restricted expression of cytotactin during development of the chicken embryo. J Cell Biol 102:1917-1930.

Crossin KL, Hoffman H, Tan S-S, Edelman GM (1989) Cytotactin and its proteoglycan ligand mark structural and functional boundaries in somatosensory cortex of the early postnatal mouse. Dev Biol 136: 381-392.

Crossin KL, Prieto AL, Hoffman S, Jones FS, Friedlander DR (1990) Expression of adhesion molecules and the establishment of boundaries during embryonic and neural development. Exp Neurol 109:618.

Epperlein HH, Halfter W, Tucker RP (1988) The distribution of fibronectin and tenascin along migratory pathways of the neural crest in the trunk of amphibian embryos. Development 103:743-756.

Faissner A (1988) Monoclonal antibody identifies a proteoglycan expressed by a subclass of glial cells. Soc Neurosci Abstr 14:920.

Faissner A, Kruse J (1990) J1/tenascin is a repulsive substrate for CNS neurons. Ncuron 5:627-637.

Gallo V, Bertolotto A (1990) Extracellular matrix of cultured glial cells: selective expression of chondroitin-4-sulfate by type-2 astrocytes and their progenitors. Exp Cell Res 187:211-223.

Goldberger M, Murray M (1974) Restitution of functional and collateral sprouting in cat spinal cord: the deafferented animal. J Comp Neurol 158:37-54.

Grierson JP, Petroski RE, Ling DSF, Geller HM (1990) Astrocyte topography and tenascin/cytotactin expression: correlation with the ability to support neuritic outgrowth. Dev Brain Res 55:11-19.

Grumet M, Hoffman S, Crossin KL, Edelman GM (1985) Cytotactin, an extracellular matrix protein of neural and nonneural tissues that mediates glia-neuron interaction. Proc Natl Acad Sci USA 82:80758079.

Hoffman S, Crossin KL, Edelman GM (1988) Molecular forms, binding functions and developmental expression patterns of cytotactin and CTB proteoglycan, an interactive pair of extracellular matrix molecules. J Cell Biol 106:519-532.

Jenkins WM, Merzenich MM (1987) Reorganization of neocortical representations after brain injury: a neurophysiological model of the basis of recovery from stroke. Prog Brain Res 71:249-266.

Kruse J, Keilhauer G, Faissner A, Timpl R, Schachner M (1985) The $\mathrm{J} 1$ glycoprotein - a novel nervous system cell adhesion molecule of the L2/HNK-1 family. Nature 316:146-148.

Lynch G, Matthews DA, Mosko S, Parks T, Cotman C (1972) Induced acetylcholinesterase-rich layer in the rat dentate gyrus following entorhinal lesions. Brain Res 42:311-318.

Mackie EJ, Tucker RP, Halfter W, Chiquet-Ehrishmann R, Epperlein IIII (1988) The distribution of tenascin coincides with pathways of neural crest cell migration. Development 102:237-250.

Maxwell WL, Follows R, Ashhurst DE, Berry M (1990a) The response of the cerebral hemisphere of the rat to injury. I. The mature rat. Philos Trans R Soc Lond [Biol] 328:479-500.

Maxwell WL, Follows R, Ashhurst DE, Berry M (1990b) The response of the cerebral hemisphere of the rat to injury. II. The neonatal rat. Philos Trans R Soc Lond [Biol] 328:501-513.

McKeon RI, Vietje BP, Wells J (1989) Increase in acetylcholinesterase in the molecular layer of the dentate gyrus in the absence of septal inputs following selective granule cell lesions. Brain Res 503:317321.

Newgreen DF, Scheel M, Kastner V (1986) Morphogenesis of sclerotome and neural crest in avian embryos: in vivo and in vitro studies on the role of notochordal extracellular matrix material. Cell Tissue Res 244:299-313.

Newgreen DF, Powell ME, Moser B (1990) Spatiotemporal changes in HNK-1/L2 glycoconjugates on avian embryo somite and neural crest cells. Dev Biol 139:100-120.

Perris R, Johansson S (1990) Inhibition of neural crest cell migration by aggregating chondroitin sulfate proteoglycans is mediated by their hyaluronan-binding region. Dev Biol 137:1-12.

Perris R, Krotoski D, Lallier T, Domingo C, Sorrell JM, Bronner-Fraser $M$ (1991) Spatial and temporal changes in the distribution of proteoglycans during avian neural crest cell development. Development 111:583-599.

Pindzola RR, Silver J (1990) The molecular environment of the dorsal root entry zone during development and after root crushes. Soc Neurosci Abstr 16:164.

Prieto AL, Jones FS, Cunningham BA, Crossin KL, Edelman GM 
(1990) Localization during development of alternatively spliced forms of cytotactin by in situ hybridization. J Cell Biol 111:685-698.

Puchala E, Windle WF (1977) The possibility of structural and functional restitution after spinal cord injury. A review. Exp Neurol 55: $1-42$.

Ramon y Cajal S (1928) Degeneration and regeneration of the nervous system. New York: Hafner.

Reier PJ, Houle JD (1988) The glial scar: its bearing on axonal elongation and transplantation approaches to CNS repair. In: Advances in neurology: functional recovery in neurological disease (Waxman SG, ed), pp 87-138. New York: Raven.

Reier PJ, Stensaas LJ, Guth L (1983) The astrocytic scar as an impediment to regeneration in the central nervous system. In: Spinal cord reconstruction (Kao CC, Bunge RP, Reier PJ, eds), pp 163-195. New York: Raven.

Rudge JS, Silver J (1990) Inhibition of neurite outgrowth on astroglial scars in vitro. J Neurosci 10:3594-3603.

Rudge JS, Smith GM, Silver J (1989) An in vitro model of wound healing in the CNS: analysis of cell reaction and interaction at different ages. Exp Neurol 103:1-16.

Savio T, Schwab ME (1989) Rat CNS white matter, but not gray matter, is nonpermissive for neuronal cell adhesion and fiber outgrowth. J Neurosci 9:1126-1133.

Schnell L, Schwab ME (1990) Axonal regeneration in the rat spinal cord produced by an antibody against myelin-associated neurite growth inhibitors. Nature 343:269-272.

Schwab ME, Caroni P (1988) Oligodendrocytes and CNS myelin are nonpermissive substrates for neurite growth and fibroblast spreading in vitro. J Neurosci 8:2381-2393.

Smith GM, Miller RH, Silver J (1986) Changing role of forebrain astrocytes during development, regenerative failure, and induced regeneration upon transplantation. J Comp Neurol 251:23-43.

Smith GM, Rutishauser U, Silver J, Miller RH (1990) Maturation of astrocytes in vitro alters the extent and molecular hasis of neurite outgrowth. Dev Biol 138:377-390.

Snider WD, Johnson EM (1989) Neurotrophic molecules. Ann Neurol 26:489-506.
Snow DM, Silver J (1990) Neurite-inhibiting proteoglycans may help determine unidirectionality of neurite growth in the developing or regenerating CNS. Soc Neurosci Abstr 16:150.

Snow DM, Lemmon V, Carrino DA, Caplan AI, Silver J (1990a) Sulfated proteoglycans present in astroglial barriers during development in vivo inhibit neurite outgrowth in vitro. Exp Neurol 109:111-130.

Snow DM, Steindler DA, Silver J (1990b) Molecular and cellular characterization of the glial roof plate of the spinal cord and optic tectum: a possible role for a proteoglycan in the development of an axon barrier. Dev Biol 138:359-376.

Stanfield B, Cowan WM (1982) The sprouting of septal afferents to the dentate gyrus after lesions of the entorhinal cortex in adult rats. Brain Res 232:162-170.

Steindler DA, Cooper NGF, Faissner A, Schachner M (1989) Boundaries defined by adhesion molecules during development of the cerebral cortex: the $\mathrm{J} 1 /$ tenascin glycoprotein in the mouse somatosensory cortical barrel field. Dev Biol 131:243-260.

Steindler DA, O'Brien TF, Laywell E, Harrington K, Faissner A, Schachner M (1990) Boundaries during normal and abnormal brain development: in vivo and in vitro studies of glia and glycoconjugates. Exp Neurol 109:35-56.

Tan SS, Crossin KL, Hoffman S, Edelman GM (1987) Asymmetric expression in somites of cytotactin and its proteoglycan ligand is correlated with neural crest cell distribution. Proc Natl Acad Sci USA 84:7977-7981

Tosney KW, Oakley RA (1990) The perinotochordal mesenchyme acts as a barrier to axon advance in the chick embryo: implications for a general mechanism of axonal guidance. Exp Neurol 109:75-89.

Verna JM, Fichard A, Saxod R (1989) Influence of glycoaminoglycans on neurite morphology and outgrowth patterns in vitro. Int $\mathbf{J}$ Dev Neurosci 7:389-399.

Zaremba S, Naegele JR, Barnstable CJ, Hockfield S (1990) Neuronal subsets express multiple high-molecular-weight cell-surface glycoconjugates defined by monoclonal antibodies CAT 301 and VC 1.1. J Neurosci 10:2985-2995. 